\title{
Wichtige endokrine Funktionsstörungen bei Schwangeren
}

Klaus Mann, Gerhard Hintze

\begin{abstract}
Der Beginn und die weitere Entwicklung einer Schwangerschaft werden durch ein fein aufeinander abgestimmtes hormonelles System der feto-plazentaren-maternalen Funktionseinheit ermöglicht. Viele Faktoren tragen dazu bei, ob der Kinderwunsch in Erfüllung geht und ob Mutter und Kind die besondere Lebensphase bis zur Geburt gesund durchleben. Besonders bei endokrinen Störungen müssen die Mütter engmaschig überwacht und ihre Therapien häufig angepasst werden.
\end{abstract}

Physiologische Hormonveränderungen I Der Beginn einer Schwangerschaft geht mit einer Vielzahl an physiologischen Hormonveränderungen einher. Bereits 7-10 Tage nach der Konzeption gelingt der Nachweis des humanen Choriongonadotropins (HCG) im Serum und ab Tag 10 im Urin mittels Schwangerschaftstests. Das Gelbkörperhormon Progesteron steigt kräftig an und große Mengen Östron und Östradiol werden sezerniert. In der 10.-12 Schwangerschaftswoche (SSW) kommt es zu einer vermehrten Schilddrüsenhormonbildung und über den hypophysär-hypothalamischen Regelkreis zu einem Abfall des TSH. Im 2. Trimenon steigt TSH wieder an, während der fT4- Spiegel sinkt [1]. Das Parathormon (PTH) steigt auf etwa das Doppelte des normalen Referenzwerts.

Endokrine Störungen | Endokrine Störungen haben einen bedeutsamen Einfluss auf die Fertilität, den Verlauf der Schwangerschaft und die fetale Entwicklung.

\section{Schilddrüse}

\section{Probleme bei Kinderwunsch}

Schilddrüsenfunktionsstörungen und -erkrankungen sind die am häufigsten diskutierte Problematik bei Frauen mit unerfülltem Kinderwunsch und sollten primär diagnostiziert oder ausgeschlossen werden ( $\triangleright$ Tab. $\mathbf{1}$ ).

Autoimmunität | Bei infertilen Frauen sind häufiger als bei Kontrollgruppen Schilddüsenperoxidaseantikörper (TPO-Ak), insbesondere in Verbindung mit einem polyzystischen Ovarial-Syndrom (PCOS) oder einer Endometriose nachzuweisen [2, 3]. Selbst bei euthyreoter Stoffwechsellage ist dabei die Fehlgeburtenrate erhöht. Weitere Autoimmunphänomene und -erkrankungen sind bei bekannter Immunthyreoiditis (Hashimoto-Thyreoiditis) in ca. $10 \%$ zu finden.
Die klinische Symptomatik ist häufig unspezifisch und die Erkrankungen bleiben lange unentdeckt.

Dies gilt vor allem für die erworbene Sprue (Gluten-sensitive Enteropathie) und den Vitamin-B12-Mangel (perniziöse Anämie). Es gibt aber auch klinisch präkonzeptionell unerkannte Fälle einer primären Nebenniereninsuffizienz, die durch deutlich erhöhte ACTH-Spiegel diagnostizierbar sind. Sehr selten gibt es eine Komorbidität mit Diabetes-mellitus Typ 1 mit eindeutiger klinischer Symptomatik. Die eingeschränkte Fertilität bei Immunthyreoiditis und dem polyendokrinen Autoimmun-Syndrom ist pathophysiologisch weitgehend ungeklärt. Die TPO-Ak-assoziierte Subfertilität scheint altersunabhängig zu sein $[4,5]$.

Hypothyreose und Fertilität | Mögliche Ursachen einer Hypothyreose vor und während der Schwangerschaft sind:

- Immunthyreoiditis (Hashimoto-Thyreoiditis)

- Z.n. Schilddrüsenoperation oder Radiojodtherapie

- sekundäre Hypothyreose (TSH und fT4 erniedrigt) bei Hypophysenerkrankungen

- iatrogene Hypothyreose unter antithyreoidaler Therapie bei Hyperthyreose

- Levothyroxinkarenz bei Hypothyreose

- Z.n. externer Strahlentherapie im Kopf- Halsbereich

Bei manifester, aber auch subklinischer Hypothyreose kommt es zu Zyklusstörungen und ovulatorischen Störungen. Postkonzeptionell besteht ein erhöhtes Risiko für Schwangerschaftskomplikationen [6]. Hierzu gehören Fehlgeburten, Frühgeburten, niedriges Geburtsgewicht, Gestationsdiabetes, arterielle Hypertonie und Präeklampsie. Ob diese Komplikationen durch eine Levothyroxin-Substitution zu minimieren sind, wird in der Literatur kontrovers diskutiert [7, 8].

Mehrbedarf an Levothyroxin bei in-vitro-Fertilisation I Im Rahmen einer in-vitro-Fertilisation mit 


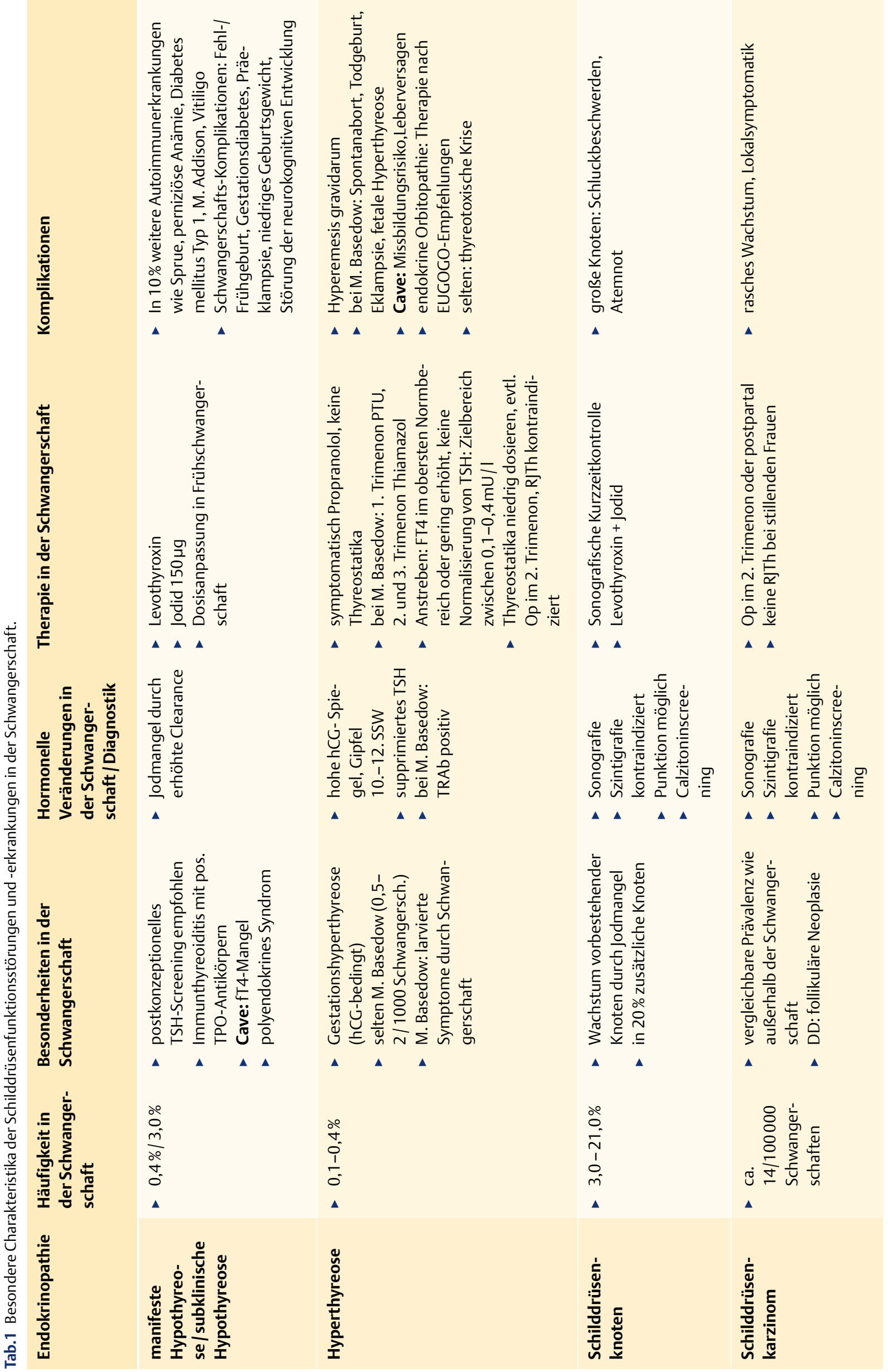


ovarieller Hyperstimulation wird Östrogen-bedingt ein Mehrbedarf an Levothyroxin induziert, der ausgeglichen werden muss. Bei zuvor noch euthyreoter Immunthyreoiditis können möglicherweise endogen nicht mehr ausreichend Schilddrüsenhormone gebildet und eine Substitution erforderlich werden.

Jodversorgung I Deutschland ist nach den Kriterien der WHO nicht mehr als Jodmangelgebiet eingestuft. Vereinzelt und regional unterschiedlich besteht dennoch ein latenter Jodmangel. Der Fortschritt wurde durch freiwillige Jodierung des Speisesalzes und breite Verwendung erreicht (Empfehlung Arbeitskreis Jodmangel). In der Schwangerschaft besteht ein erhöhter Jodbedarf durch die erhöhte Jodclearance.

Die WHO empfiehlt deshalb eine tägliche Jodzufuhr von $250 \mu \mathrm{g} /$ Tag während der Schwangerschaft und Stillzeit.

Bei der derzeitigen Jodversorgung wird eine zusätzliche Jodsupplementation von $150 \mu \mathrm{g} / \mathrm{Tag}$ zur ausreichenden Versorgung des Feten und Neugeborenen empfohlen. Diese Dosierung ist auch bei Patientinnen mit Autoimmunthyreopathien (Hashimoto-Thyreoiditis, M. Basedow) gerechtfertigt. Verwendet werden derzeit meist nicht-rezeptpflichtige Kombinationspräparate bestehend aus Jod $150 \mu \mathrm{g}$, Folat $800 \mu \mathrm{g}$ bis Ende der 12. SSW-Woche und Folat $400 \mu \mathrm{g}$ ab der 13. SSW sowie optional allen B-Vitaminen oder selektiv Vitamin B12, ergänzt durch Vitamin D3 800 IE und der Omega-3- Fettsäure DHA $200 \mathrm{mg}$ (Folio forte ${ }^{\circledR}$, Folio-D3 ${ }^{\circledR}$, femibion $1^{\circledR}$ und femibion $2^{\circledR}$ ).

Selenmangel und Selentherapie I Die allgemeine Selenversorgung ist in Deutschland nicht optimal [9]. Die Selenkonzentration im Serum war in der LIANCO-Kohorte mit $68 \pm 32 \mu \mathrm{g} / \mathrm{l}(\mathrm{n}=792)$ und in der EPIC-Kohorte mit $66 \mu \mathrm{g} / \mathrm{l}(\mathrm{n}=202$ männliche Teilnehmer) im Vergleich zu erwünschten Serumspiegeln von 101-139 $\mu$ g/l erniedrigt. Valider zur Beurteilung des Selenstatus wäre die allerdings nicht allgemein verfügbare Messung der Selenoproteine. Eine ausreichende Versorgung wird in der Schwangerschaft und post Partum angestrebt [10]. Selenoproteine sind am antioxidativen Schutz des Schilddrüsengewebes beteiligt. Da die Glutathionperoxidase und Selenoprotein P (SelP) nur bei ausreichender Selenversorgung ihre maximale Konzentration und Aktivität erreichen, kann ein Mangel über eine $\mathrm{H}_{2} \mathrm{O}_{2}$-Anreicherung im Schilddrüsengewebe zu Gewebeschäden beitragen und entzündliche Prozesse fördern. Klinische Studien haben gezeigt, dass die Prävalenz der Immunthyreoiditis und Hypothyreose bei Serumspiegeln um $100 \mu \mathrm{g} / 1$ signifikant niedriger als bei Selenmangel ist (Odds-Ratio 0,47-0,75, [10]). Die Wirksamkeit einer Selensubstitution mit $200 \mu \mathrm{g}$ täglich wurde bei der Postpartum-Thyreoiditis gezeigt [11]. Es entwickelten sich weniger manifeste Hypothyreosen, postpartale Schilddrüsenfehlfunktionen und ein Abfall der Schilddrüsenperoxidase-Antikörper. Auch die klinische Aktivität der endokrinen Orbitopathie und die Befindlichkeit der Patienten war mit $200 \mu g$ Selen (Natriumselenit) im Vergleich mit Placebo bzw. Pentoxifyllin über 6 Monate günstig zu beeinflussen [12].

\section{Hyperthyreose}

Eine manifeste Hyperthyreose ist mit einer Prävalenz von $0,1-0,4 \%$ in der Schwangerschaft selten. In der Regel handelt es sich bei jungen Frauen um eine immunogene Hyperthyreose (M. Basedow ), erst im höheren Lebensalter um eine funktionelle Autonomie bei Knotenstruma und selten eine disseminierte Autonomie. Anamnestisch ist eine Überdosierung mit Levothyroxin (Hyperthyreosis factitia) zu klären. Die zweithäufigste Ursache ist die Gestationshyperthyreose. Der M. Basedow mit oder ohne endokrine Orbitopathie tritt in 0,5-2 / 1000 Schwangerschaften auf.

Differenzialdiagnostisch sind Morbus Basedow und Gestationshyperthyreose durch die TRAb-Bestimmung eindeutig zu trennen.

Morbus Basedow I Der M. Basedow beginnt meist mit klassischen Symptomen wie Ruhetachykardie, innerer Unruhe, Hyperhidrosis, Wärmeintoleranz, Haarausfall, Schlaflosigkeit, Gewichtsabnahme oder Durchfall. Zyklusstörungen treten erst bei schweren Verlaufsformen auf. Die Schilddrüse ist häufig nicht vergrößert, jedoch stark durchblutet ( $\triangleright$ Abb. 1 ), so dass Strömungsgeräusche auskultierbar und ein Strumaschwirren tastbar werden können. Die endokrine Orbitopathie wird klinisch entsprechend dem Clinical Activity Score der europäischen Arbeitsgruppe EUGOGO erfasst [13].

Gestationshyperthyreose I Die Symptomatik der Gestationshyperthyreose ist milde und durch die der Frühschwangerschaft überlagert. So sind die Ruhetachykardie, vermehrtes Schwitzen, Befindlichkeitsstörungen, Haarausfall und Schlaflosigkeit kaum verwertbar. Meist liegt eine Grenzwerthyperthyreose im 1. Trimenon vor. Als manifeste Hyperthyreose ist sie häufig mit einer Hyperemesis gravidarum vergesellschaftet. Entscheidend ist daher die Labordiagnostik. Neben fT4, fT3 und TSH muss der TSH- Rezeptor-Antikörper (TRAb) gemessen werden, der negativ bleibt. Die Schilddrüsensonografie zeigt nur bei der immunogenen Hyperthyreose die diffuse Hypervaskularisation (Gefäßinferno) und typische Echoarmut. Bei Mehrlingsschwangerschaften können die HCG-Spiegel besonders hoch anstei- 
gen und eine Gestationshyperthyreose begünstigen. Eine thyreostatische Therapie verbietet sich. Im Einzelfall kann für einige Wochen eine kurzzeitige, symptomatische Therapie mit einem BRezeptorenblocker (Propranolol) versucht werden. Bei längerer Anwendung kann es zu fetalen Wachstumsstörungen kommen [14].

Therapie der Autoimmunhyperthyreose I Liegt ein manifester M. Basedow präkonzeptionell vor, raten wir von einer Schwangerschaft ab und empfehlen eine sichere Kontrazeption bis zur Remission.

Bei dringendem Kinderwunsch ist eine frühzeitige, ablative Therapie durch near total Thyreoidektomie oder Radiojodtherapie zu empfehlen.

Die anschließende Levothyroxin-Substitution muss optimiert und unter Kontrolle sein. Präsentiert sie sich in der Schwangerschaft als manifeste Hyperthyreose, besteht eine akute Gefährdung für Mutter und Kind. Es besteht ein erhöhtes Risiko für Spontanaborte, Todgeburten, Eklampsie, Herzinsuffizienz bis hin zur lebensbedrohlichen thyreotoxischen Krise $[15,16]$. Durch die Costimulation von HCG im 1. Trimenon ist die Hyperthyreose in dieser vulnerablen Phase der fetalen Organentwicklung häufig besonders stark ausgeprägt, im 2. Trimenon kommt es zur klinischen und immunologischen Beruhigung und postpartal nicht selten zur Reaktivierung.

TSH-Rezeptor-Antikörper bestimmen I TSH-Rezeptor-Antikörper (TRAb) sind plazentagängig und können durch Stimulation des fetalen TSHRezeptors eine fetale Hyperthyreose (1-5\%) [13] und Struma connata hervorrufen. Selten werden auch blockierende TRAb wirksam, die dann sehr selten zu einer fetalen und neonatalen Hypothyreose führen können. Am Ende des 2. Trimenons sollte TRAb bestimmt werden, wenn zu diesem Zeitpunkt

- eine thyreostatische Therapie erforderlich ist,

- Operation oder Radiojodtherapie vorangegangen sind oder

- in einer früheren Schwangerschaft eine neonatale Hyperthyreose aufgetreten ist.

Die intrauterine Sonografie ist für die Beurteilung der fetalen Schilddrüse, einer Wachstumsretardierung oder Hydrops, die Echokardiografie für Pumpfunktion als Herzinsuffizienzzeichen und Herzfrequenzanalyse wichtig. Eine enge Kooperation zwischen Gynäkologen, Endokrinologen und peripartal Neonatologen ist wichtig, um fetale Komplikationen zu verhindern.

Die Hyperthyreose ist mit Risiken wie Frühgeburtlichkeit, geringem Geburtsgewicht und konnatalen Anomalien belastet.

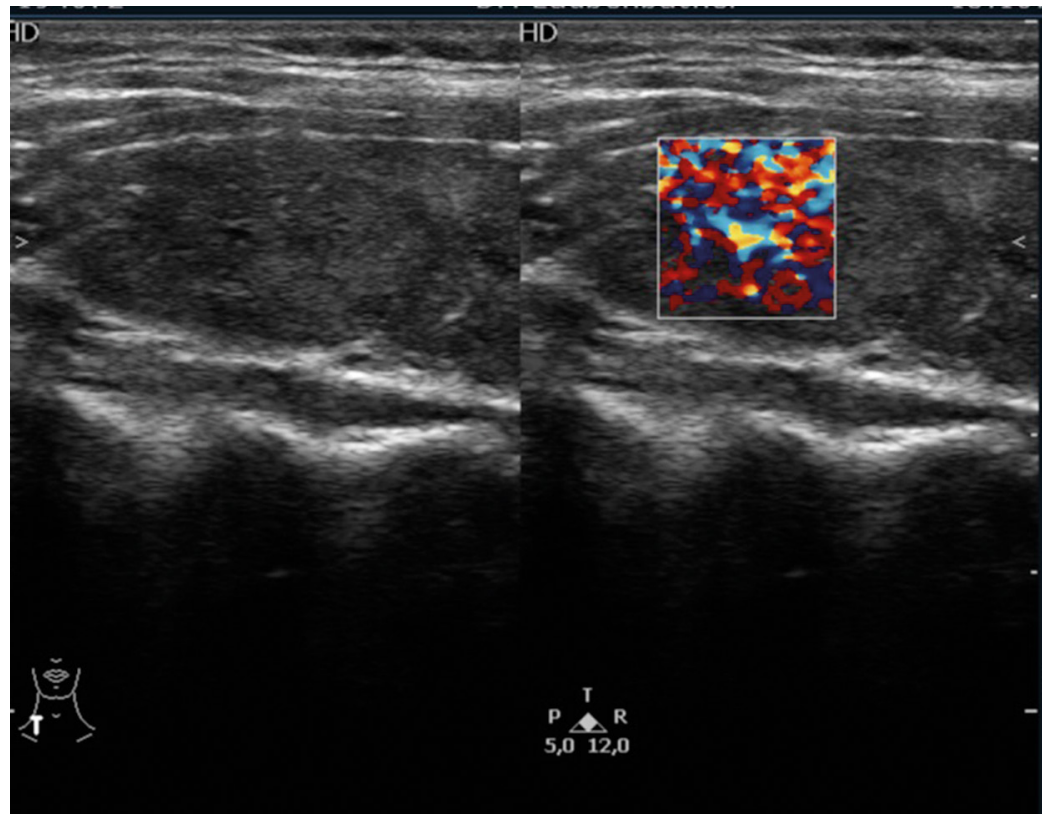

Abb. 1 Florider Morbus Basedow in der Schwangerschaft, Schilddrüsensonografie mit ausgeprägter Gefäßhyperperfusion (Gefäßinferno).
Übertherapie vermeiden I Die thyreostatische Therapie sollte in möglichst niedriger Dosierung eingesetzt, klinisch, sonografisch und laborchemisch engmaschig kontrolliert werden. Sie soll einerseits die Risiken einer unbehandelten Hyperthyreose minimieren, andererseits bei milden Formen eine Übertherapie vermeiden, die zu einer Hypothyreose und connatalen Struma führen kann. Ziel der Behandlung ist ein Absenken von fT4 in den obersten Trimenon-spezifischen Referenzbereich, allerdings in Kenntnis der methodischen Assayprobleme von Analog-Tracer-Methoden in der Schwangerschaft. Zusätzlich können fT3 und im Zweifelsfall zusätzlich GesamtT4 (TT4) gemessen werden. Eine Normalisierung von fT4, fT3 und vor allen TSH gilt als Übertherapie und muss durch Dosisreduktion vermieden werden. Monatliche klinische, sonografische und laborchemische Kontrollen sind erforderlich. Eine Kombinationstherapie mit Levothyroxin ist kontraindiziert [15].

Zeitpunkt der Therapie | Die Diskussion, welches Thyreostatikum zu welcher Zeit eingesetzt werden soll, ist weiter im Fluss und wird wegen des Nebenwirkungsprofils der beiden Medikamente Methimazol (MMI) und Propylthiouracil (PTU) unterschiedlich bewertet. Derzeitiger Konsens ist im 1. Trimenon PTU zu verwenden und $3 \times$ tgl. wegen der kurzen Halbwertzeit zu geben. Die Dosis beträgt $3 \times 50 \mathrm{mg}$ mit evtl. Steigerung auf $3 \times 100 \mathrm{mg}$. Die Empfehlung basiert auf der Beobachtung seltener Missbildungen wie Aplasia cutis, Choanalatresie, Ösophagusatresie durch MMI. PTU ist belastet durch akutes Leberversagen (1/10000 Erwachsenen, 1/2000 Kinder, mittlere Dosis $300 \mathrm{mg} / \mathrm{Tag}$ ) zwischen Tag 6-450. Die routinemäßige Kontrolle von Leberwerten kann dies nicht verhindern. Eine zeitliche Begrenzung auf die Zeit der Organogenese erscheint sinnvoll. Im 
Anschluss, d.h. im zweiten und dritten Trimenon, sollte auf MMI umgesetzt werden, das wegen der längeren Halbwertzeit nur einmal tgl. gegeben werden muss.

Nebenwirkungen | Beiden Präparaten sind etwa vergleichbar die bekannten allergisch-toxischen Nebenwirkungen zu eigen. Hierzu gehören Hautreaktionen, Arthralgien, gastrointestinale Symptome und z.T. lebensbedrohlich eine Panzytopenie oder Agranulozytose (0,35-0,4\%). Bei PTU finden sich häufiger als bei MMI eine Vaskulitis und antineutrophile Antikörper sowie ausschließlich die immunallergische Hepatitis (0,1-0,2\%) [16]. Die empfohlene Anfangsdosierung von MMI liegt bei $10 \mathrm{mg}$, bei hoher Jodbelastung $20 \mathrm{mg}$ tgl. mit rascher Dosisreduktion auf 5 bzw. 2,5 mg. Angestrebt werden TSH-Spiegel nicht über $0,5 \mathrm{mIU} / \mathrm{ml}$. Häufig kann im Verlauf ganz abgesetzt werden. Da Carbimazol durch endogene Esterasen in MMI verwandelt wird, sind Wirkung und Nebenwirkungsprofil vergleichbar (15 mg Carbimazol entsprechen $10 \mathrm{mg}$ Thiamazol). Eine Operation ist mit Abortrisiko und vorzeitiger Entbindung belastet. Sie ist nur sehr selten bei medikamentös nicht beherrschbarer Hyperthyreose (Cave Non-Compliance) und/oder visusbedrohender endokriner Orbitopathie, Krankheitspersistenz mit hohem Thyreostatikumverbrauch oder sehr großer Struma indiziert. Der beste Zeitpunkt ist das 2. Trimenon, in dem der Uterus für eine Weheninduktion am resistentesten ist. Eine Radiojodtherapie ist streng kontraindiziert.

\section{Hypothyreose}

TSH-Screening vor und während der Frühschwangerschaft | Aufgrund der widersprüchlichen Datenlage über fetale Folgeschäden, insbesondere neuropsychologischer Entwicklungsstörungen [17], bei einer unerkannten und nicht frühzeitig behandelten manifesten, aber auch latenten Hypothyreose empfehlen internationale Leitlinien (noch) kein generelles TSH-Screening in der Frühschwangerschaft. Ein Screening basierend auf Risikofaktoren und anamnestischen Angaben, die die Wahrscheinlichkeit einer Hypothyreose in der Schwangerschaft erhöhen sollen, nimmt allerdings eine erhebliche Dunkelziffer unbehandelter Patienten in Kauf. Da Störungen der Gehirnentwicklung schon in den ersten Schwangerschaftswochen auftreten und eine frühe Substitution davon abgeleitet werden kann [18], unterstützen die Autoren die aktuelle europäische Leitlinie, die ein generelles TSH-Screening postkonzeptionell befürwortet. Ziel sollte es sein, diese Empfehlung in den Mutterpass und in die Mutterschaftsrichtlinien aufzunehmen.

Risiko für Mutter und Kind | Eine aktuelle Metaanalyse der Mayoklinik zur Bedeutung der sub- klinischen Hypothyreose [19] belegt das erhöhte Risiko für Aborte (Relatives Risiko [RR] 2,01; Konfidenzintervall [KI] 1,66-2,44), Plazentariss (RR 2,14; KI 1,23-3,70), vorzeitigen Blasensprung (RR 1,43; KI 1,04-1,95) und sogar neonatalen Tod (RR 2,58; KI 1,41-4,73). Überraschenderweise gab es darin eine vergleichende Studie mit oder ohne Levothyroxingabe, in der kein Nutzen der Substitution hinsichtlich Abort, vorzeitiger Geburt, Schwangerschaftshypertonie, niedrigem Geburtsgewicht oder niedrigem Apgar-Score nachzuweisen war [19]. Diesbezüglich bleibt die Datenlage demnach widersprüchlich.

Frühzeitige Substitution sinnvoll | Da eine niedrig dosierte, am TSH orientierte Levothyroxingabe keine wesentlichen Nebenwirkungen hat, überwiegen aus Sicht der Autoren die Argumente für eine frühzeitige Substitution. Bei einer Gabe von $50 \mu$ g Levothyroxin in den ersten Wochen nach Bekanntwerden eines positiven Schwangerschaftstests, zeitnahem TSH-Screening und einer Kontrolle von TSH und fT4 nach 4 Wochen sowie der TPO-Ak, besteht praktisch kein Risiko einer Überdosierung mit möglichen negativen Folgen auf die Hirnentwicklung wie sie in der Rotterdamer Studie „Generation R“ beschrieben wurden [20]. Mehrere Studien haben zudem gezeigt, dass eine subklinische Hypothyreose zu einer Beeinträchtigung der neurokognitiven Entwicklung führt [17, 21]. Die Untersuchungen von Haddow et al. [21] wurden im Alter von 7 Jahren mit verschiedenen anerkannten neurophsychologischen Tests durchgeführt. Diesen Ergebnissen widerspricht die Arbeit von Lazarus et al. [22], die keine derartige Störungen beobachten konnten. Der Unterschied ist wahrscheinlich dadurch zu erklären, dass in letzerer Arbeit die Levothyroxingabe in der Verumgruppe zu spät, und zwar erst in der 12. SSW nach Abschluss der Gehirnentwicklung erfolgte und die Kinder im Alter von 3 Jahren getestet wurden, in dem die Validierung der neuropsychologischen Tests zweifelhaft bleibt.

Erhöhtes Risiko für Aufmerksamkeits-DefizitHyperaktivitäts-Syndrom (ADHS) | Kritisch zu würdigen ist die Teilstudie der Niederländischen „Generation R“ Studie von Ghassabian et al. [23]. Die Autoren konnten zeigen, dass auch nach Korrektur der TSH-Variablen in der Multivariatanalyse (logistische Regression) erhöhte maternale TPO-Ak (147 Frauen [4,7\%] mit Titern über $100 \mathrm{U} / \mathrm{ml}$ ) zwar keine Vorhersage für verbale und nicht-verbale kognitive Funktionen (Language Development Survey und Parent Report of Children's Ability) im Alter von 2,5 Jahren erbringen konnten, jedoch ein deutlich erhöhtes Risiko für ein ADHS bestand (RR 1,56; KI 1,14-2,14). Dies zeigt, dass zerebrale, noch nicht verstandene Prozesse bei der Schilddrüsenautoimmunität ablaufen. 
TSH-Spiegel

TSH >2,5 mU/I bei Fertilitätsabklärung

TSH $>2,5 \mathrm{mU} / \mathrm{l}$ in der Frühschwangerschaft:

$\mathrm{TSH}>3,0 \mathrm{mU} / \mathrm{lim} 2$. Trimenon

$\mathrm{TSH}>3,5 \mathrm{mU} / \mathrm{lim}$ 3. Trimenon

\section{Therapie}

Bestimmung von fT4 und TPOAk, bei erhöhtem TSH Levothyroxin $50 \mu \mathrm{g} \mathrm{tgl.,}$ Dosisanpassung nach 4 Wochen

sofort Levothyroxin $50 \mu \mathrm{g}$ tgl., Dosisanpass. nach 4 Wo.

Levothyroxin $50 \mu \mathrm{g} \mathrm{tg}$.

Levothyroxin $50 \mu \mathrm{g} \mathrm{tg}$.

Bei Krankheitsbeginn kann eine geringe Hyperthyreose auftreten, die keiner thyreostatischen Behandlung bedarf, die aber die Differenzialdiagnose zum M. Basedow erschwert, da auch bei der Immunhyperthyreose die TPO-Ak erhöht sind. Die TRAb-Bestimmung klärt aufgrund der hohen Spezifität für den M. Basedow die Situation. Allerdings gibt es auch Übergänge beider Autoimmunerkrankungen mit einem Wechsel des Antikörperprofils, der klinischen Symptome und der biologischen Aktivität der TSH-Rezeptorantikörper von stimulierend zu blockierend.

nerapie mit Selen, deren Wirksamkeit postpartal belegt ist [40]. Diese Untersuchung entkräftet aber nicht die Empfehlung eines allgemeinen TSH-Screenings, da auch in der genannten Studie eine TSH-Erhöhung ein gesicherter negativer Einflussfaktor war (RR 1,77; KI 1,15-2,72). Auch tierexperimentelle Befunde sprechen dafür, dass das fetale zentrale Nervensystem bei einer Hypothyroxinämie irreversibel geschädigt wird. Historische Bilder von Kretins ziehen die Parallele dazu (mentale Retardierung, motorische Entwicklungsstörungen, Sprach- und Hörstörungen).

Diagnostik der Hypothyreose | Die Prävalenz der manifesten Hypothyreose liegt in der Schwangerschaft bei $0,4 \%$ und für die subklinische (latente) Hypothyreose bei $3 \%$. Die Diagnose wird durch erhöhte TSH-Werte (außerhalb des Trimenonspezifischen Referenzbereichs) und erniedrigtes fT4 (manifest) oder normales fT4 (subklinisch) gestellt.

Für die ursächlich am häufigsten vorkommende Immunthyreoidits ist die Bestimmung der Schilddrüsenperoxidase-Antikörper (TPOAk $>50-100$ U/l, 80-90\% positiv) obligat.

Seltener sind auch die Thyreoglobin-Antikörper (Tg-Ak) gleichzeitig oder bei negativen TPO-Ak selektiv erhöht. Die Schilddrüsensonografie zeigt fast immer den typischen Befund einer kleinen, meist unter $6 \mathrm{ml}$ großen, inhomogenen, diffus oder auch regional echoarmen Schilddrüse (atrophische Variante) mit initial duplexsonografisch erfassbarer mäßiger Hyperperfusion. Bei länger dauernder Erkrankung ist das atrophische Schilddrüsengewebe meist minderdurchblutet.

Erschwerte Differenzialdiagnose I Die klassische Hashimoto-Thyreoiditis in seiner hypertrophen Form (Volumen $>18 \mathrm{ml}$ ) ist in Deutschland selten.
Keine typischen Symptome I Typische Symptome finden sich nicht bei der subklinischen Hypothyreose, und selbst die manifeste Hypothyreose kann durch unspezifische Symptome der Frühschwangerschaft maskiert werden. Hierzu gehören Kloßgefühl, Übelkeit, Haarausfall, Stimmungslabilität, depressive Verstimmung, Ödeme u.a.m.). Die Immunthyreoiditis kann als polyendokrines Syndrom mit anderen Autoimmunerkrankungen kombiniert sein (ca. 10\%). Hierzu gehören

- der M. Addison (primäre Nebenniereninsuffizienz bei Immunadrenalitis [24],

- die Zöliakie (Glutenunverträglichkeit) und

- die klinisch einfach erfassbare Vitiligo.

Selten kommen der sich rasch manifestierende Diabetes mellitus Typ 1 und ein primärer Hypoparathyreoidismus vor. Zeiten einer hormonellen Umstellung sind für die Aktivierung dieser Autoimmunprozesse prädestiniert. ist es, rasch eine euthyreote Stoffwechsellage zu erreichen. Der Handlungskorridor von Schilddrüsenspezialisten orientiert sich an nationalen und internationalen Leitlinien wie z.B. der Endocrine Practice Guideline der amerikanischen Endocrine Society [15]. Bei bekannter, substituierter Hypothyreose muss präkonzeptionell die Levothyroxindosis optimiert und nach 4 Wochen überprüft werden. In der Frühschwangerschaft ist eine Dosisanpassung erforderlich. Bei etwa $80 \%$ der Frauen ist eine Dosissteigerung von $30-50 \%$ erforderlich. Sie richtet sich nach den Trimenonspezifischen Referenzbereichen von TSH. Die Empfehlungen sind in $>$ Tab. 2 zusammengefasst. Der Hormonbedarf steigt bereits ab der 4. SSW wie oben dargestellt und macht eine Dosissteigerung von Levothyroxin um 30-50\% erforderlich. Ist die Dosierung angepasst, sollten am Ende des
Therapie der Hypothyreose | Ziel der Behandlung
Tab. 2 Anpassungsschema zur Levothyroxin-Substitution in der Schwangerschaft. 
1. Trimenons und in der 20. SSW TSH und fT4 kontrolliert werden. Ein gut praktikabler Weg der Dosissteigerung besteht darin, die bisherige Dosis um 2 Tabletten Levothyroxin/Woche von der Patientin selbst steigern zu lassen, sobald sie über die eingetretene Schwangerschaft Kenntnis hat. Bei Athyreose und schon präkonzeptionell hohen Levothyroxindosen muss die Adaptation subtiler durch den Spezialisten gesteuert werden. Bei Vorliegen eines polyendokrinen Syndroms sind die anderen Autoimmunerkrankungen fachspezifisch zu behandeln.

\section{Schilddrüsenknoten}

Schilddrüsenszintigrafie ausgeschlossen | Die Abklärung und Therapie von Schilddrüsenknoten in der Schwangerschaft unterscheidet sich nicht wesentlich vom Vorgehen außerhalb der Schwangerschaft. Kontraindiziert ist eine Schilddrüsenszintigrafie und Radiojodtherapie. Die Verschlechterung der Jodversorgung durch die erhöhte Jodidclearance kann in Jodmangelgebieten das Wachstum vorbestehender Knoten stimulieren und neue Knoten induzieren. Eine dabei vorliegende relevante uni- oder multifokale Autonomie ist ohne Szintigrafie nicht eindeutig zu sichern. Die wichtigsten Fragen sind in den Guidelines der American Thyroid Association beantwortet und nach Evidenzgrad bewertet worden [25].

Aktuelle Studienlage | In Gebieten eines moderaten Jodmangels finden sich bei 3-21\% Schilddrüsenknoten, die bei Nullipara signifikant seltener (9,4\%) als bei Mehrgebärenden sind (1: 20,7\%, 2 : 20,7\%, 3: 33,9\%). In einer belgischen Studie verdoppelte sich die Knotengröße bei $60 \%$ der Knoten, sie blieben jedoch in der Größe zwischen 5 und 12 Millimeter. Wurde ein Knoten im 1. Trimenon entdeckt, entwickelten sich bei bis zu $20 \%$ zusätzliche Knoten im Laufe der Schwangerschaft. Mit höherem Lebensalter verstärkt sich das Problem. Die Prävalenz von Schilddrüsenkarzinomen (überwiegend papilläre Karzinome, PTC) war im kalifornischen Krebsregister mit 14,4/ 100000 vergleichbar zu der außerhalb der Schwangerschaft. Die Diagnostik stratifiziert das Vorgehen nach dem Ultraschallbefund, der Anamnese und dem klinischen Untersuchungsbefund. Eine aktuelle Übersicht einschließlich Feinnadelpunktion ist im Deutschen Ärzteblatt zu finden [26]. Es besteht kein Konsens über die routinemäßige Bestimmung von Calcitonin zum Ausschluss oder Beleg eines medullären Schilddrüsenkarzinoms. Außerhalb der Schwangerschaft wird die Bestimmung zunehmend empfohlen.

Schilddrüsensonografie und Feinnadelpunktion | Die größte Bedeutung haben sonografische Kriterien, die die Sensitivität und Spezifität der Malignomdiagnostik erhöhen. Hierzu gehören
Echoarmut, Mikrokalzifikationen, unscharfe Randbegrenzung und zentrale Hyperperfusion. Mit Hilfe der noch nicht als Standardverfahren etablierten Elastografie wird die Rigidität des Knotens beurteilt. Karzinome weisen häufig einen höheren Härtegrad (Sensitivität 86\%, Spezifität $87 \%$ ) als benigne Knoten auf. Elastografisch weiche Knoten sind praktisch nie maligne. Damit besteht ein sehr hoher negativer Vorhersagewert. Die Punktion von Schilddrüsenknoten und Lymphknoten hat kein erhöhtes Morbiditätsrisiko [26].

Therapie I Im seltenen Fall eines zytologisch diagnostizierten Schilddrüsenkarzinoms (PTC) oder einer gesicherten Indikation zur Operation bei unklarer, follikulärer Neoplasie empfielt sich die Thyreoidektomie im 2. Trimenon oder erst postpartal, da sich die Prognose hierdurch nicht verschlechtert und eine zusätzliche Radiojodablation dann möglich ist. In der Stillzeit ist die Radiojodtherapie allerdings ebenfalls kontraindiziert. Nach einer notwendigen Radiojodtherapie wird ein Konzeptionsschutz für 6-12 Monate empfohlen. Die posttherapeutische Levothyroxindosierung soll wie außerhalb der Schwangerschaft einen Zielwert für TSH um 0,5 mIU/l erreichen. Im 2. Trimenon ist für Mutter und Kind das OP-Risiko am geringsten. Ein mögliches Wachstum des Tumors und/oder von Lymphknoten sollte durch Sonografie und die Bestimmung von Thyreoglobulin alle 6-12 Wochen beobachtet werden. In dieser Beobachtungsphase wird in den Guidelines der amerikanischen Schilddrüsengesellschaft (ATA) eine niedrig dosierte Levothyroxingabe empfohlen (Zielwert für TSH 0,1-1,5 mU/L, [25]) Der Algorithmus zur Knotenabklärung und -therapie in der Schwangerschaft und post partum ist den ATA- Guidelines zu entnehmen.

\section{Nebennierenrindeninsuffizienz}

Selten aber folgenschwer | Werden während einer Schwangerschaft anhaltende Übelkeit, eine ungewöhnliche Müdigkeit oder eine Hypotonie beobachtet, so muss differenzialdiagnostisch eine mögliche, noch nicht bekannte Nebennierenrindeninsuffizienz bedacht werden. Hierbei handelt es sich zwar um ein seltenes Ereignis, dies kann jedoch dramatische Folgen nach sich ziehen [27, 28]. Als „Goldstandard“ wird bei entsprechendem Verdacht der Kortikotropin-Test (ACTH-Test) empfohlen [27]. Ist eine Nebennierenrindeninsuffizienz nicht ausgeschlossen, kann eine probatorische Hydrokortisonmedikation vorgenommen werden [28].

Besonderheiten bei bekanntem M. Addison | Die im Kontext der Schwangerschaft relevanten Erkrankungen der Nebenniere umfassen M. Addison und das Adrenogenitale Syndrom ( $\bullet$ Tab. 3). 


\begin{tabular}{|c|c|c|c|c|c|}
\hline $\begin{array}{l}\text { Endokrinopa- } \\
\text { thie }\end{array}$ & $\begin{array}{l}\text { Häufigkeit in } \\
\text { der Schwanger- } \\
\text { schaft }\end{array}$ & $\begin{array}{l}\text { Besonderheiten in } \\
\text { der Schwangerschaft }\end{array}$ & $\begin{array}{l}\text { Hormonelle Verände- } \\
\text { rungen in der Schwan- } \\
\text { gerschaft / Diagnostik }\end{array}$ & $\begin{array}{l}\text { Therapie in der } \\
\text { Schwangerschaft }\end{array}$ & Komplikationen \\
\hline $\begin{array}{l}\text { primäre } \\
\text { Nebennieren- } \\
\text { rindeninsuffizi- } \\
\text { enz }\end{array}$ & - & $\begin{array}{l}\text { meist Autoimmun- } \\
\text { adrenalitis } \\
\text { hohe Krisengefähr- } \\
\text { dung }\end{array}$ & $\begin{array}{l}\text { 21-Hydroxylase- } \\
\text { Antikörper positiv } \\
\text { ACTH erhöht, } \\
\text { Urincortisol } \\
\text { vermindert }\end{array}$ & $\begin{array}{l}\text { Hydrocortison } \\
\text { 15-25 mg/Tag } \\
\text { - Fludrokortison } \\
\text { 0,1-0,2 mg/pro } \\
\text { Tag } \\
\text { - kein Dexame- } \\
\text { thason! }\end{array}$ & $\begin{array}{l}\text { polyendokrines } \\
\text { Syndrom: bei } \\
\text { gleichzeitiger } \\
\text { Hypothyreose } \\
\text { immer zuerst } \\
\text { Hydrocortison, } \\
\text { erst dann } \\
\text { Levothyroxin } \\
\text { peripartal } \\
100 \text { mg Hydro- } \\
\text { cortison }\end{array}$ \\
\hline $\begin{array}{l}\text { adrenogenitales } \\
\text { Syndrom }\end{array}$ & $\begin{array}{l}\text { 1:5000- } \\
\text { 1:15000 bei } \\
\text { Neugebore- } \\
\text { nenscreening } \\
\text { Heterozygote } \\
\text { Genträger } \\
\text { ca. } 2 \%\end{array}$ & $\begin{array}{l}\text { klassisch mit } \\
\text { Salzverlustsyn- } \\
\text { drom, hypotone } \\
\text { Dehydratation } \\
\text { - neugeborene } \\
\text { Mädchen mit } \\
\text { virilisierten } \\
\text { äußeren Genitalien }\end{array}$ & $\begin{array}{l}\text { 21-Hydroxylase- } \\
\text { mangel bei } 95 \% \\
\text { 11-Hydroxylase- } \\
\text { mangel bei } 5 \% \\
\text { - erhöhte Cortisol- } \\
\text { werte durch CBG } \\
\text { bedingt } \\
\text { - Renin erhöht } \\
\text { - Elektrolyte, } \\
\text { Steroidhormonana- } \\
\text { lysen }\end{array}$ & $\begin{array}{l}\text { wie bei } \\
\text { Nebennieren- } \\
\text { rindeninsuffizi- } \\
\text { enz } \\
\text { bei Wehenbe- } \\
\text { ginn erhöhte } \\
\text { Stressdosis }\end{array}$ & - \\
\hline
\end{tabular}

Tab. 3 Besondere Charakteristika der Erkrankungen der Nebenniere in der Schwangerschaft.
Zur Diagnose und Therapie der primären Nebennierenrindeninsuffizienz (M. Addison) wurden kürzlich neue Leitlinien der Endocrine Society publiziert [27]. Sie behandeln auch das Vorgehen in der Schwangerschaft.

Bei adäquater Überwachung können auch Patientinnen mit M. Addison eine normale Schwangerschaft und einen normalen Geburtsverlauf haben. Insofern kann bei bestehendem Kinderwunsch nicht von einer Schwangerschaft abgeraten werden.

Bei Frauen mit primärer Nebennierenrindeninsuffizienz liegt - wie bei anderen Autoimmunerkrankungen auch - eine etwas eingeschränkte Fertilität, eine leicht erhöhte Rate für Spontanaborte und für eine Frühgeburt vor $[29,30]$.

Auf Symptome achten | Während der Schwangerschaft sind Frauen mit einem M. Addison engmaschig zu überwachen. Hierbei ist auf Symptome zu achten, wie

- ungewöhnliche allgemeine Schwäche,

- ausgeprägte Müdigkeit,

- hypotensive Blutdruckwerte oder

- Symptome einer möglichen Hyperglykämie.

Hierdurch sollen Phasen einer zu niedrigen oder zu hohen Substitutionsdosis erfasst werden. Da es physiologischerweise während einer Schwangerschaft zu einem Anstieg der Kortisolkonzentration im Serum kommt [30], muss die Substitutionsdosis von Hydrokortison während der
Schwangerschaft angepasst werden. Dies ist spätestens ab der 24. Schwangerschaftswoche erforderlich, wobei in der Regel eine Erhöhung um wenigstens $20 \%$, eher $40-50 \%$ der Hydrokortisondosis ausreichend ist. Ausschlaggebend ist das klinische Befinden der Schwangeren.

Dexamethason vermeiden | Die genannten Empfehlungen [27] sprechen sich für die Verabreichung von Hydrokortison aus, nicht von Kortisonazetat, Prednisolon oder Prednison. Insbesondere Dexamethason sollte unbedingt vermieden werden, da es in der Plazenta nicht deaktiviert wird und somit auf den fetalen Kreislauf übertreten kann. Häufig ist zudem eine Erhöhung der Fludrokortisondosis indiziert, als Folge des Antimineralokortokoideffektes von Progesteron [28].

Aus diesem Grund sollten während der Schwangerschaft regelmäßig Kontrollen der Elektrolytwerte vorgenommen werden.

Auch ein Abfall des Blutdrucks kann ein Symptom eines Mineralokortikoidmangels sein.

Dosis während und nach der Geburt | Während der Entbindung sind Hydrokortisondosen von $100 \mathrm{mg}$ als Bolus, gefolgt von $200 \mathrm{mg} / 24$ Stunden i.v. zu applizieren [27]. Beschrieben wurde eine höhere Sektiorate [31]. Nach der Entbindung und nach Erholung der Frau kommt es in der Regel zu einer raschen Normalisierung, so dass dann auf die ursprüngliche Substitutionsdosis für Hydro- 


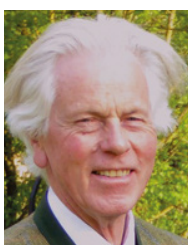

Prof. Dr. med. Klaus Mann ist em. Professor der Klinik für Endokrinologie und Stoffwechselerkrankungen der Universität DuisburgEssen, Leiter des Endokrinologie-Zentrums Alter Hof Gemeinschaftspraxis Alter Hof, München, sowie im Schilddrüsenzentrum Tegernsee tätig. klaus.mann@ezah.de www.ezah.de

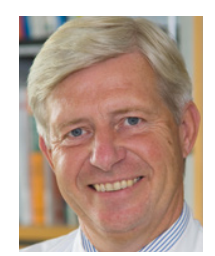

Prof. Dr. med. Gerhard Hintze

ist ehemaliger Chefarzt der Abteilung für Innere Medizin, Asklepios Klinik Bad Oldesloe. Heute arbeitet er als niedergelassener Internist in der Praxisgemeinschaft „Auf dem Meere" in Lüneburg. GHintze@t-online.de kortison und Fludrokortison reduziert werden kann [27]. Andere empfehlen eine Verdoppelung der Erhaltungsdosis während der ersten 24-48 Stunden post partum [28]. Generell gilt, dass sowohl die betroffenen Patientinnen, ihre Angehörigen als auch die behandelnden Ärzte und Hebammen mit der Überwachung der Schwangeren während der Schwangerschaft und während der Entbindung vertraut sein müssen.

Während der Schwangerschaft und bei der Entbindung muß die Glukokortikoiddosis erhöht werden. Wichtig ist eine individuelle Schulung über Notsituationen und das Mitführen eines Notfallausweises.

\section{Vitamin D}

Wirkung bleibt offen | Während einer Schwangerschaft besteht nicht selten ein Vitamin-D-Mangel. Immer wieder wurde auch vermutet, dass aus einem möglichen Vitamin-D-Mangel ein erhöhtes Risiko für Schwangerschaftskomplikationen, wie ein erhöhtes Frühgeburtsrisiko oder ein zu geringes Wachstums des Feten, resultieren kann [32]. Eine kürzlich veröffentlichte Metaanalyse ging dieser Frage weiter nach [33]. Die Autoren fassen zusammen, daß eine Vitamin D- Supplementierung im Vergleich zu einer Plazebogruppe zwar zu einem signifikanten Anstieg von 25-Hydroxy-Vitamin D führt, die Resultate aber inkonsistent seien. So wurde nur in zwei der sieben untersuchten randomisierten Studien ein niedrigeres Präeklampsierisiko nach Vitamin-D-Substitution berichtet (8,9\% vs. 15,5\%). Zwei andere Studien konnten dies hingegen nicht bestätigen.

Insgesamt muss somit ein positiver Effekt einer Vitamin-D- Supplementierung auf den Verlauf der Schwangerschaft offen bleiben [34].
Konsequenz für Klinik und Praxis

- Physiologische Hormonveränderungen der Schilddrüse, Nebennieren und des Kalziumstoffwechsels sind in der Schwangerschaft, aber auch prä- und postnatal zu beachten.

- Die Fertilität ist auch bei subklinischer Hypothyreose beeinträchtigt und sollte bei Kinderwunsch behandelt werden.

- Ein generelles TSH-Screening in der Frühschwangerschaft und eine Jodsubstitution mit $150 \mu \mathrm{g} \mathrm{tgl.} \mathrm{in} \mathrm{der} \mathrm{Schwangerschaft}$ und Stillzeit wird auch bei vorbestehender Autoimmunthyreoiditis empfohlen.

- Eine unbehandelte manifeste, aber auch subklinische Hypothyreose kann zu Störungen der fetalen, neurokognitiven Entwicklung führen. Eine frühzeitige Substitution und Dosiserhöhung von Levothyroxin bei vorbehandelter Hypothyreose erscheint trotz widersprüchlicher Datenlage aus Sicherheitsgründen gerechtfertigt.

- Eine manifeste und subklinische Hyperthyreose hat ein erhöhtes Abortrisiko und muss diferenzialdiagnostisch abgeklärt werden. Der M. Basedow (TRAb-positiv) soll niedrig dosiert thyreostatisch behandelt und engmaschig im Verlauf kontrolliert werden. Die hCG-bedingte Gestationshyperthyreose (TRAb-negativ) darf nicht antithyreoidal und nur passager mit Propranolol therapiert werden.

- Die Abklärung von Schilddrüsenknoten unterscheidet sich nicht wesentlich von der außerhalb der Schwangerschaft. Eine Tc-Szintigraphie und Radiojodtherapie ist streng kontraindiziert, eine Schilddrüsenpunktion problemlos möglich. Bei zytologischem Verdacht oder Beleg eines Schilddrüsenkarzimons (meist PTC) ist eine OP am besten im 2. Trimenon, aber auch nach der Entbindung vertretbar.

- An eine Nebenniereninsuffizienz sollte bei anhaltender Übelkeit, ausgeprägter Müdigkeit, Vitiligo, Hypotonie und Elektrolytverschiebungen gedacht und ein ACTH-Kurztest durchgeführt werden. Die Substituion mit Hydrocortison muss höher gewählt werden und mit Fludrocortison bei Hypotonie ergänzt werden.

\section{Interessenkonflikt}

Die Autoren geben an, dass kein Interessenkonflikt besteht.

DOI 10.1055/s-0042-111582 Dtsch Med Wochenschr 2016; 141: 1304-1312 (c) Georg Thieme Verlag KG . Stuttgart · New York . ISSN 0012-0472
Literatur

1 Führer D. Schilddrüsenerkrankungen und Schwangerschaft. Der Internist 2011; 52: 1158-1166

2 Poppe K, Velkeniers B, Glinoer D. The role of autoimmunity in fertility and pregnancy. Nat clin prac Endocr Metab 2008; 4: 394-405

3 Janssen OE, Mehlmauer N, Hahn S et al. High prevalence of autoimmune thyroiditis in patients with polycystic ovary syndrome. Eur J Endocrinol 2004; 150: 363-369

4 Thanagartian S. Tan A, Knox E at al. Association between thyroid autoantibodies and misscariage and preterm birth: meta-analysis of evidence. BMI 2011; 342: 2616-2624

Vollständiges Literaturverzeichnis unter http://dx.doi.org/10.1055/s-0042-111582 
5 Bullmann C. Minnemann, T. Schilddrüse, Fertilität und Schwangerschaft. Der Gynäkologe 2015; 48: 537-546

6 Glinoer $\mathrm{D}$. The regulation of thyroid function in pregnancy: pathways of endocrine adaption from physiology to pathology. Endocr Rev 1997; 14: 194-202

7 Wasserstrum N, Anania CA. Prenatal consequences of maternal hypothyroidism in early pregnancy and inadequate replacement. Clin Endocrinol 1995; 42: 353-358,

8 Stagnaro-Green A, Pearce E. Thyroid disorders in pregnancy. Nature Reviews Endocrinology 2012; 8: 650-658

9 Stoffaneller R, Morse NL. A review of diatary selenium intake and selenium status in Europe and the middle East. Nutrience 2015; 7: 1494-1537

10 Wu Q, Rayman MP, Lv H et al. Low population selenium status is associated with increased prevalence of thyroid disease. I Clin Endocrinol Metab 2015; 100: 4037-4047

11 Negro R, Greco G, Mangieri T et al. The influence of selenium supplementation on postpartum thyroid status in pregnant women with thyroid peroxidase autoantibodies. JCEM 2007; 92: 1263-1268

12 Marcocci C, Kahaly GL, Krassas GE et al. Selenium and the course of mild Graves orbitopathy. New Engl J Med 2011; 364: 1920-1931

13 Bartalena L, Baldeschi L, Boboridis K et al. The 2016 European Thyroid Association / European group on Graves' orbitopathy guideline fort he managemenet of Graves 'orbitopathy. Europ Thyroid J 2016; 5: 9-26

14 Azizi F, Amouzegar A. Management of hyperthyroidism during pregnancy and lactation. Europ Endocrinol 2011; 164: 871-876

15 Abalovich, M, Amino N, Barbour LA et al. Management of thyroid dysfunction during pregnancy and postpartum : an endocrine society clinical practice guideline. J Clin Endocrinol Metab 2007; 92: S1S47

16 Krassas GE, Poppe K, Glinoer D. Thyroid function and human reproductive health. Endocr Rev 2010; 31: 702-755

17 Perres-Lobato R, Ramos R, Arrebola JP, Calvente I et al. Thyroid status and its association with cognitive functioning in healthy boys at 10 years of age. Eur J Endocrinol 2015; 172: 129-139

18 Führer D, Mann K, Feldkamp J et al. Schilddrüsendysfunktion in der Schwangerschaft. Dtsch Med Wschr 2014; 139: 2148-2152

19 Maraka S, Ospina NM, O‘Keeffe DT. Subclinical Hypothyroidism in Pregnancy: A Systematic Review and Meta-Analysis. Thyroid 2016; 26: 580-590

20 Korevaar TI, Muetzel R, Medici M et al. Association of maternal thyroid function during early pregnancy with offspring IQ and brain morphology in childhood: a population-based prospective cohort study. Lancet Diabetes Endocrinol 2016; 4: 35-43
21 Haddow JE, Palomaki GE, Allan WC et al. Maternal thyroid deficiency during pregnancy and subsequent neuropsychological development oft he child. N Engl J Med 1999; 341: 549-855

22 Lazarus J, Brown RS, Daumerie C et al. 2014 European thyroid association guidelines for the management of subclinical hypothyroidism in pregnancy and in children. Eur Thyroid ] 2014; 3: 76-94

23 Ghassabian A, Bongers-Schokking J], de Rijke YB et al. Maternal thyroid autoimmunity during pregnancy and the risk of attention deficit / hyperactivity problems in children: the Generation $\mathrm{R}$ Study. Thyroid 2012; 22: 178-186

24 Pulzer A, Burger-Stritt S, Hahner S. Morbus Addison Der Internist 2016, 5: 457-467

25 Stagnaro-Green A, Abalovich M, Alexander E et al. Guidelines of the American thyroid association for the diagnosis and management of thyroid disease during pregnancy and postpartum. Thyroid 2011, 21: 1081-1116

26 Feldkamp J, Führer D, Luster M, Musholt T], Spitzweg C, Schott M. Feinnadelpunktion in der Abklärung von Schilddrüsenknoten. Deutsches Ärzteblatt 2016, 113: 353-359

27 Bornstein SR, Allolio B, Arlt W et al. Diagnosis and Treatment of Primary Adrenal Insufficiency: An Endocrine Society Clinical Practice Guideline. J Clin Endocrinol Metab 2016; 101: 364-381

28 Lebbe M, Arlt W. What ist the best diagnostic and therapeutic management strategy for an Addison patient during pregnancy? Clin Endocrinol 2013; 78: 497-502

29 Erichsen MM, Husebye ES, Michelsen TM et al.. Sexuality and fertility in women with Addison's disease. J.Clin.Endocrinol.Metab. 2010; 95: 4354-43607

30 Jung C, Ho JT, Torpy TJ et al. A longitudinal study of plasma and urinary cortisol in pregnancy and post partum. J Clin Endocrinol Metab 2011; 96 : 1533-1540

31 Remde H, Zopf K, Schwander J, Quinkler M. Fertility and pregnancy in primary adrenal insufficiency in Germany. Horm Metab Res 2016; 48: 306-311

32 Miliku K, Vinkhuyzen A, Blanken LM et al. Maternal vitamin $\mathrm{D}$ concentrations during pregnancy, fetal growth patterns, and risks of adverse birth outcomes. Amer ] Clin Nutr 2016; pii: ajcn 123752

33 Palacios C, De-Regil LM, Lombardo LK, Pena-Rosas JP. Vitamin D supplementation during pregnancy: updated meta-analysis on maternal outcomes. J. Steroid. Biochem Mol Biol 2016; doi: 10.1016/j.jsbmb.2016.02.008

34 De-Regil LM, Palacios C, Lombardo LK, Pena-Rosas JP. Vitamin D Supplementation for women during pregnancy. Cochrane Database Syst Rev 2016; 14: doi: $10.1002 / 14651858 . C D 008873$ 